\title{
Liberales Verständnis
}

\section{Carlos Quinto}

Dr. med., Mitglied des FMH-Zentralvorstandes, Departementsverantwortlicher Public Health und Gesundheitsberufe

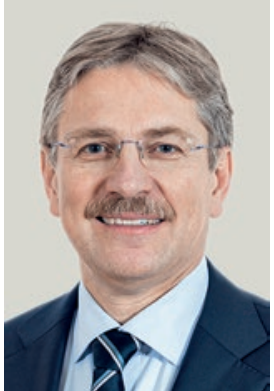

Wir sind Angehörige eines freien Berufs. Diese Freiheit benötigen wir, um die grosse Varianz der Problemstellungen, mit denen uns Patientinnen und Patienten in unserer täglichen Praxis aufsuchen, einer Lösung zuführen zu können. Diese Lösungen werden idealerweise gemeinsam mit den Patientinnen und Patienten erarbeitet. Denn Evidence-Based Medicine beinhaltet drei Komponenten: Beste verfügbare wissenschaftliche Evidenz, unsere klinischen Fähigkeiten und Erfahrung sowie die Werte und Vorstellungen der Patientinnen und Patienten. Wir benötigen ebenfalls Freiheiten, um eine Arzt-Patienten-Beziehung in einem geschützten Rahmen stattfinden lassen zu können. Nur in einer vertrauensvollen Umgebung sind Patientinnen und Patienten bereit und in der Lage, uns gewisse wesentliche Informationen im Anamnesegespräch zu geben. Rahmenbedingungen sind folglich essentiell. Staatliche Rahmenbedingungen sollten unsere Tätigkeit erleichtern und nicht zunehmend erschweren und ineffizient werden lassen. Wir behandeln in erster Linie Menschen und nicht Papier.

Nicht zufällig hat der Schweizer Philosoph Peter Bieri je ein Buch den Themen Freiheit und Würde gewidmet. Es bestehen starke Wechselwirkungen mit unserer Gesundheit. Kinder und Jugendliche sollen gefördert werden, auch in ihrer Gesundheit. Dies ist ein Kernanliegen der Initiative «Kinder ohne Tabak». Die Initiative wird von der ganzen Ärzteschaft getragen und von der FMH entsprechend dem Beschluss der Ärztekammer

\section{Nachweislich richtet sich Tabakwerbung oft} gezielt an Jugendliche und sie wirkt. Wo es mehr Werbung hat, rauchen mehr Jugendliche.

unterstützt. Wir sind als freier Beruf sozial engagiert und liberal eingestellt. Es entspricht aber nicht unserem liberalen Verständnis, Minderjährige gezielt in die Nikotinabhängigkeit zu führen. Die wissenschaftliche Evidenz dafür, dass die entsprechende Werbung aber genau diese Wirkung hat und sich gezielt an Jugendliche richtet, ist erdrückend.

Es ist nicht konsequent, den Verkauf an unter 18-Jährige zu verbieten und diese Altersgruppe weiterhin gezielt zu bewerben. Dies ist aber für die Tabakindustrie der effizienteste Weg, «Langzeitkunden» zu generieren, um die Verstorbenen zu ersetzen. Denn wer als Minderjähriger mit dem Nikotinkonsum beginnt, ist schneller, stärker und länger abhängig, und das zeitlebens. Diese wissenschaftlichen Fakten sind auch der Tabakindustrie seit langem bekannt und werden voll ausgenutzt. Nachdem der Ständerat 2019, notabene unter liberaler Führung, einen soliden Gesetzesentwurf geschrieben hatte, wurde dieser im Nachgang vom Nationalrat total verwässert. Hinsichtlich Kinder- und Jugendschutz ist der aktuelle «Gegenentwurf» ein Ge-

Liebe Kolleginnen und Kollegen, bitte stimmen sie am 13. Februar "JA» zur Initiative "Kinder ohne Tabak».

setz für das Landesmuseum. Alle Werbeformate, die sich gezielt an Minderjährige richten, sind weiterhin erlaubt und ein paar alte, für Kinder und Jugendliche aus der Mode gekommene (nur 0,2\% der Werbeausgaben) werden verboten. Die Gewinne streichen ausländische Grosskonzerne ein. Mit dem aktuellen Gegenvorschlag des Tabakproduktegesetzes werden sich bei uns neue, für Minderjährige attraktive Nikotinapplikatoren auf dem Markt durchsetzen. Dies weil sie günstig sind und man mit ihnen Nikotin hochdosiert konsumieren kann. In China, wo diese Applikatoren produziert werden, ist ihre Anwendung übrigens per Gesetz verboten. Ein entsprechender Artikel wurde letztes Jahr in der SÄZ publiziert. Die neue Evidenz ist in der Politik leider noch nicht angekommen. Aktuell bezahlt jede vierköpfige Schweizer Familie, egal ob Raucher oder Nichtraucher, CHF 1500 Krankenkassenprämien pro Jahr für die Folgen des Rauchens. Man sollte bei der Ursache ansetzen, anstatt unser Gesundheitswesen mit Kostenzielen und Globalbudgets zu deckeln: Die Leute krank zu machen und sie dann nicht zu behandeln entspricht nicht unserem Berufsethos. Ich bitte Sie also, liebe Kolleginnen und Kollegen, stimmen Sie am 13. Februar "JA» zur Initiative «Kinder ohne Tabak» entsprechend unserer Berufung und unserem Berufseid, Gesundheit zu schützen. Was die Tabakprävention anbelangt, sollten wir nicht länger europäisches Schlusslicht bleiben. Auch wir wollen die Raucherprävalenz bei Minderjährigen um 10 Prozent senken können, wie das andere europäische Länder geschafft haben. 